\title{
Nanostructure-Enhanced Surface Acoustic Waves Biosensor and Its Computational Modeling
}

\author{
Guigen Zhang, 2,3 \\ ${ }^{1}$ Department of Bioengineering, Clemson University, Clemson, SC 29634, USA \\ ${ }^{2}$ Department of Electrical and Computer Engineering, Clemson University, Clemson, SC 29634, USA \\ ${ }^{3}$ Institute for Biological Interfaces of Engineering, Clemson University, Clemson, SC 29634, USA
}

Correspondence should be addressed to Guigen Zhang, guigen@clemson.edu

Received 29 December 2008; Revised 1 May 2009; Accepted 23 June 2009

Recommended by Wojtek Wlodarski

\begin{abstract}
Surface acoustic wave (SAW) devices are considered to be very promising in providing a high-performance sensing platform with wireless and remote operational capabilities. In this review, the basic principles of SAW devices and Love-mode SAW-based biosensors are discussed first to illustrate the need for surface enhancement for the active area of a SAW sensor. Then some of the recent efforts made to incorporate nanostructures into SAW sensors are summarized. After that, a computational approach to elucidate the underlying mechanism for the operations of a Love-mode SAW biosensor with nanostructured active surface is discussed. Finally, a modeling example for a Love-mode SAW sensor with skyscraper nanopillars added to in its active surface along with some selected results is presented.
\end{abstract}

Copyright (C) 2009 Guigen Zhang. This is an open access article distributed under the Creative Commons Attribution License, which permits unrestricted use, distribution, and reproduction in any medium, provided the original work is properly cited.

\section{Introduction}

Biosensors are analytical devices that combine a biologically sensitive element with a physical transducer to selectively and quantitatively discern specific compounds in a given biological environment [1]. Biosensors play a crucial role in safeguarding public health and protecting the environment. Due to the ever-increasing concerns over public health and environmental safety, improving the performance of biosensors becomes not just a technological issue but rather issues of life and death and quality of life. High performance biosensors are required to detect, specifically and sensitively, biological species that can cause harm to humans at a concentration range well below the harmful threshold level. Ideally, such biosensors should be built upon a wireless platform with which detections can be made through remote and wireless operations.

Surface acoustic wave (SAW) based biosensors have the potential to meet these requirements because their excitation and data communication can be achieved via a wireless means [2]. SAW-based sensors are microelectromechanical systems in which high frequency acoustic waves travel close to the surface of a piezoelectric substrate. Because of the confinement of the acoustic energy near the surface within the range of one acoustic wavelength, SAW devices are highly sensitive for discerning any surface perturbation such as molecular absorption or adsorption and change of viscoelastic properties.

To use a SAW device as a sensor, very often a twoport delay-line configuration is used. In such a two-port delay-line SAW sensor, two sets of interdigitated transducers (IDTs) placed atop of a piezoelectric substrate are necessary for generating and receiving acoustic waves. The first set of IDTs is called the transmitter and the second set the receiver. The transmitter converts an alternating electrical signal into an acoustic wave which travels along the surface of the piezoelectric substrate, and the receiver converts the acoustic wave back to an electrical signal for detection and analysis. The area between the generator and receiver is often coated with a chemically sensitive surface for molecular absorption or adsorption. SAW sensors can be used to detect molecular absorption and adsorption events or changes in the viscoelastic properties of the sensitive surface by measuring the wave characteristics such as frequency shift and insertion loss.

SAW devices have been widely used as sensors for gaseous, chemical, and biological species detection [3-10]. 


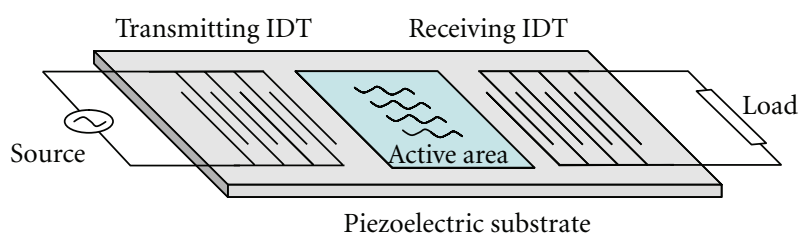

(a)

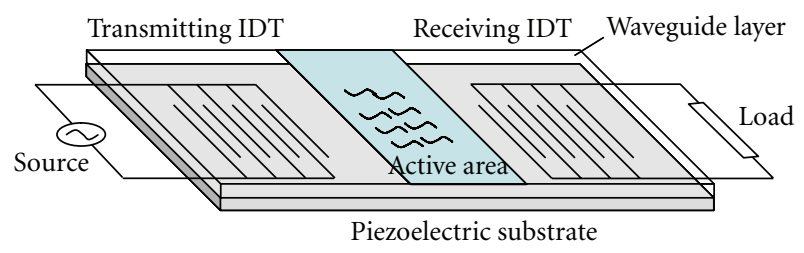

(b)

Figure 1: Schematic representations of a two-port delay-line SAW sensor (a) and a Love-mode SAW sensor (b).

The underlying principles for their operations and applications have been widely discussed and these discussions can be found in several books [2,11-13]. Recently, the applications of SAW devices as biosensors were summarized by Länge et al. [14] and their applications as gas and vapor sensors were summarized by Ippolito et al. [15]. In light of this, the focus of this review will be on the use of nanostructures to enhance the detection performances of SAW sensors. We will begin by briefly laying out the basic operating principles of a SAW device and a Love-mode SAW sensor to present the need for surface enhancement in a Love-mode SAW sensor. We will then summarize some recent efforts in incorporating nanostructures into SAW biosensors. After that, we will discuss a computational approach to elucidate the underlying mechanism of Love-mode SAW biosensors incorporated with nanostructures. Finally, we will present a modeling example of a Love-mode SAW sensor incorporated with skyscraper nanopillars along with some selective results.

\section{The Need for Enhancing the Active Area of a Love-Mode SAW Sensor}

Acoustic waves are mechanical waves generated by the displacements of atoms in a solid piezoelectric material such as quartz, lithium niobate and lithium tantalite. When an alternating mechanical strain is applied to a piezoelectric substrate, the atoms of the piezoelectric material will be displaced from their original locations against the elastic forces that tend to restore them. This action results in a mechanical wave propagating close to the surface of the material [16].

In a common two-port delay-line configuration, a SAW sensor consists of two transducers placed on the surface of the piezoelectric substrate as illustrated in Figure 1(a). The left transducer (the transmitter) is for generating a mechanical wave and the right transducer (the receiver) is for picking up the wave. The transmitter is used to convert an alternating electrical excitation into acoustic wave in the solid piezoelectric material. The generated acoustic wave travels along the surface of the piezoelectric material where the biological detection occurs. By a reverse process, the receiver converts the acoustic wave back to electrical signals. In such a two-port delay-line biosensor the dimensions of the IDTs determine the wavelength of the acoustic wave: $\lambda=2\left(W_{\mathrm{el}}+W_{\mathrm{sp}}\right)$ where $W_{\mathrm{el}}$ is the width of each individual electrode in the IDEs and $W_{\text {sp }}$ the spacing between two adjacent individual electrodes. The frequency of operation is governed by $f_{0}=v_{0} / \lambda$, where $\lambda$ is the wavelength of the acoustic wave and $v_{0}$ is the travel velocity of the acoustic wave in the piezoelectric material $[2,11-13]$.

In order to achieve high sensitivity in SAW sensors, it is essential to confine a maximum amount of acoustic energy near the surface of the substrate and minimize wave scattering into the bulk of the substrate. To achieve this, Love-mode acoustic wave devices have been developed based on the physical effect discovered by Love [17]. In Lovemode SAW devices, a waveguide layer (see Figure 1(b)) made of a dielectric material is used to confine acoustic energy close to the surface of the devices. For high waveguide efficiency the coated material should have a wave velocity less than that of the base piezoelectric substrate material [13]. Dielectric materials such as silicon dioxide, parylene, polymethylmethacrylate, photoresists, novolac resin are good waveguide materials.

Aside from trapping a maximal amount of acoustic energy close to the active surface of a SAW sensor, increasing the area of the active surface can lead to an increased amount of molecular absorption or adsorption. To provide a large detection range and high detection sensitivity, it would be ideal to have a sufficiently large surface area for the active region such that a larger amount of molecular absorption or adsorption at the active region can be achieved. Based on a brief survey of the state of SAW sensor development and application, we proposed some strategies for enhancing the performances of SAW sensors by taking advantage of nanotechnology, especially adding nanostructures to the active area of SAW sensors in 2006 [18] based on the belief that nanostructures would increase the specific surface area due to their high surface to volume ratio. Indeed, nanostructures such as nanoparticles, nanotubes, nanofibers, nanorods, and nanopillars have been successfully employed for this purpose in the past several years.

\section{Efforts in Applying Nanostructures to the Active Area of SAW Sensors}

The nanostructures added to the active surface of SAW sensors can be categorized mainly into four groups: (1) dispersed nanoparticles and nanoparticle conjugates, (2) random nanofibers, nanobelts, and nanotubes, (3) standing nanostructures, and (4) porous nanostructures. In the following sections, we will discuss these efforts according to these categories.

3.1. Nanoparticles and Nanoparticle Conjugates. Levit et al. [19] used a SAW sensor to detect toluene vapors after coating the sensor with fluoroalkyl acrylate (FAA) polymeric 
TABLE 1: Material constants for a XY-lithium-niobate piezoelectric substrate.

\begin{tabular}{lc}
\hline \multicolumn{3}{c}{ XY lithium niobate } \\
\hline$C_{11}$ & $20.3 \times 10^{10} \mathrm{~N} \cdot \mathrm{m}^{-2}$ \\
$C_{12}$ & $5.3 \times 10^{10} \mathrm{~N} \cdot \mathrm{m}^{-2}$ \\
$C_{13}$ & $7.5 \times 10^{10} \mathrm{~N} \cdot \mathrm{m}^{-2}$ \\
$C_{14}$ & $0.9 \times 10^{10} \mathrm{~N} \cdot \mathrm{m}^{-2}$ \\
$C_{33}$ & $24.5 \times 10^{10} \mathrm{~N} \cdot \mathrm{m}^{-2}$ \\
$C_{44}$ & $6.0 \times 10^{10} \mathrm{~N} \cdot \mathrm{m}^{-2}$ \\
$e_{15}$ & $3.7 \mathrm{C} \cdot \mathrm{m}^{-2}$ \\
$e_{22}$ & $2.5 \mathrm{C} \cdot \mathrm{m}^{-2}$ \\
$e_{31}$ & $0.2 \mathrm{C} \cdot \mathrm{m}^{-2}$ \\
$e_{33}$ & $1.3 \mathrm{C} \cdot \mathrm{m}^{-2}$ \\
$\varepsilon_{11}$ & 44 \\
$\varepsilon_{33}$ & 29 \\
$\rho$ & $4600 \mathrm{~kg} \cdot \mathrm{m}^{-3}$ \\
\hline
\end{tabular}

nanoparticles ( $\sim 100 \mathrm{~nm}$ diameter). The obtained sensitivity results were compared with those obtained with SAW sensors coated with the same polymer without nanoparticles. They found that a single layer coating consisting of packed polymer nanoparticles provided a surface area approximately three times greater than that of a film without nanoparticles. When exposed to toluene at a concentration of $3100 \mathrm{ppm}$, the SAW sensors coated with FAA containing nanoparticles exhibited three times greater frequency shift than SAW sensors coated with FAA without nanoparticles. Chiu and Gwo [20] showed a linear dependence of frequency shift upon surface mass density of colloidal Au nanoparticles ( $10 \mathrm{~nm}$ diameter) using a dual delay-line Rayleigh SAW sensor (built on a $\mathrm{LiNbO}_{3} 128^{\circ} \mathrm{YX}$ substrate) in a dry environment. The linear relationships reveal that the higher the mass density of Au nanoparticles the higher the frequency shift is. Moreover, they also performed detection of oligonucleotides by conjugating the oligonucleotides with Au nanoparticles in reference with a control device having just Au nanoparticles using a Love-mode SAW device (built on a $\mathrm{LiTaO}_{3} 36^{\circ}$ YX substrate) in an aqueous environment. They showed that frequency shift depends on the concentration of $\mathrm{Au}$ nanoparticles used to bind the oligonucleotides. Based on their results, they estimated an "atto gram mass standard" for the sensitivity of their nanoparticles enhanced SAW sensors. In another study, the same group applied a similar approach using Rayleigh SAW devices built on AlN substrates [21] to detect oligonucleotides conjugated with Au nanoparticles. They showed a clear change in insertion loss as well as in resonant frequency as the experimental procedure progresses from surface functionalization, noncomplimentary reactions, to after hybridization. Au colloidal nanoparticles have also been mixed with sol gel containing titania and spin-coated onto the active surface of SAW sensors fabricated on $\mathrm{LiNbO}_{3} 64^{\circ} \mathrm{YX}$ substrates [22] for hydrogen gas detection. Higher-frequency shifts were observed at $1 \% \mathrm{H}_{2}$ in a temperature range from 150 to $300^{\circ} \mathrm{C}$ for the $\mathrm{SAW}$ sensors coated with titania containing Au nanoparticles than their counterparts coated with only titania.
3.2. Random Nanofibers, Nanobelts, and Nanotubes. Of the techniques using random nanofibers to enhance the active surface of SAW sensors, conductive polymers such as polyaniline and polypyrrole are popular choices for the materials because they can be polymerized by an electrochemical means. Wu et al. [23] coated a layer of polyaniline nanofibers as the selective layer for relative humidity detection using a dual delay-line SAW system built on a $\mathrm{LiNbO}_{3} 128^{\circ}$ YX substrate. The use of polyaniline nanofibers renders the SAW sensor responsive to humidity change in a close-tolinear relationship. Sadek et al. [24] used a polyaniline/ $\mathrm{In}_{2} \mathrm{O}_{3}$ nanofiber composite to improve the sensing performance of SAW sensors for $\mathrm{H}_{2}, \mathrm{NO}_{2}$, and $\mathrm{CO}$ detection. Good repeatability was obtained for $\mathrm{H}_{2}$ and $\mathrm{CO}$ gases. For $\mathrm{NO}_{2}$, the repeatability was not as good due possibly to the poisoning of the sensitive composite by the $\mathrm{NO}_{2}$ gas at high concentrations. In a recent study, Sadek et al. [25] used a polyaniline $/ \mathrm{WO}_{3}$ nanofiber composite-based SAW devices built on a $\mathrm{LiNbO}_{3} 64^{\circ} \mathrm{YX}$ substrate with a $\mathrm{ZnO}$ waveguide layer for hydrogen gas detection at room temperature. The developed sensors showed good repeatability and a closeto-linear relationship (especially at high $\mathrm{H}_{2}$ concentrations) between hydrogen concentration and frequency shift. In a similar approach, Atashbar et al. [26] applied polyaniline nanofibers on top of an AlN waveguide layer of a SAW sensor fabricated on $\mathrm{LiNbO}_{3} 64^{\circ} \mathrm{YX}$ substrate for hydrogen detection at room temperature.

Polypyrrole is another conducting polymer often used. IL-Mashat et al. [27] developed a SAW gas sensor with polypyrrole nanofibers as the active sensing layer. They demonstrated sensitive responses of the sensors to $\mathrm{H}_{2}$ and $\mathrm{NO}_{2}$. Aside from these conducting polymer nanifibers, $\mathrm{ZnO}$ nanobelts have also been used as the active layer in a SAW sensor [28]. In this study, Sadek et al. deposited a layer of $\mathrm{ZnO}$ nanobelts on the active surface of a SAW sensor and demonstrated sensitive responses of the sensor to gases at a high temperature: $\mathrm{H}_{2}$ at $185^{\circ} \mathrm{C}$ and $\mathrm{NO}_{2}$ at $160^{\circ} \mathrm{C}$. Interesting enough, the dose-response calibration curve for $\mathrm{H}_{2}$ exhibited almost a linear relationship while the curve for $\mathrm{NO}_{2}$ was nonlinear.

Of all the nanofiber cases discussed earlier, the main purpose for using these nanostructures, including polyaniline nanofibers, polyaniline/oxide composites, polypyrrole nanofibers, and $\mathrm{ZnO}$ nanobelts, is to make the SAW sensors responsive to their intended targets such as humidity or various gases. However, the benefit of using these nanofibers or nanobelts is clearly visible from one common feature of these SAW sensors: they all showed rapid responses to their intended targets owing possibly to the high specific areas of these nanostructures. One critical issue associated with multitarget sensors, though, is their specificity: It is imperative that they be able to differentiate various different targets.

Penza et al. explored the coating of a layer of singlewalled carbon nanotubes (SWCNTs) and multiwalled carbon nanotubes (MWCNTs) onto commercially available 433.92 MHz two-port SAW resonators for vapor detection [29-31]. In general, SWCNT produced more shift in wave frequency than did MWCNT. For SAW sensors coated with SWCNT, large frequency shifts for low concentrations of 


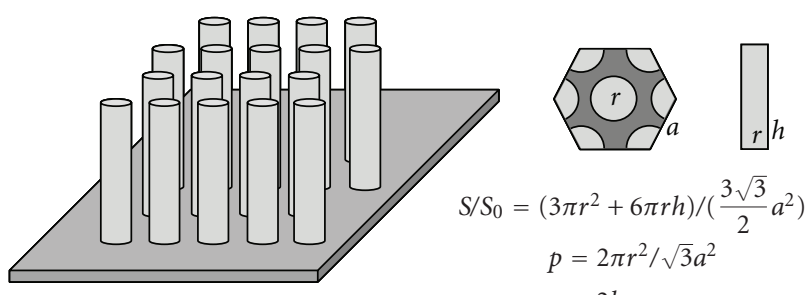

For $r=150 \mathrm{~nm}, h=6 \mu \mathrm{m}$ and $p=75 \%: S / S_{0}=\left(1+\frac{2 h}{r}\right) p=60.75$

(a)

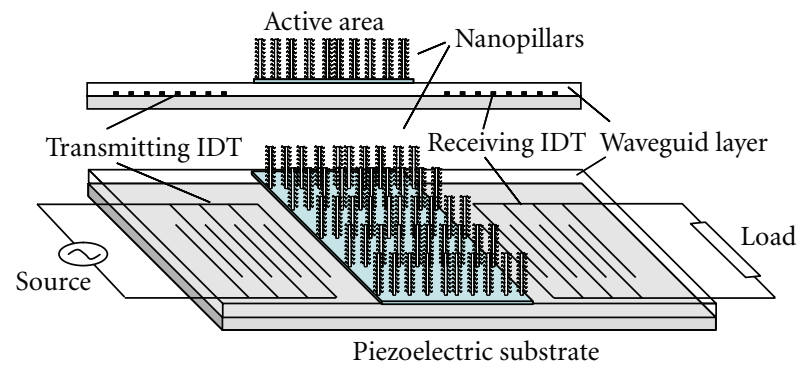

(b)

Figure 2: An illustration for the increase in surface area due to the cylindrical walls of the nanopillars (a), and a schematic representation of a two-port delay-line Love-mode SAW sensor with standing nanopillars added to the active area.

ethanol $(720 \mathrm{KHz}$ for $86 \mathrm{ppm})$ and ethylacetate $(655 \mathrm{KHz}$ for $178 \mathrm{ppm}$ ) were noted, and for sensors coated with MWCNT frequency shifts of $150 \mathrm{KHz}, 160 \mathrm{KHz}, 110 \mathrm{KHz}$ for $86 \mathrm{ppm}$ ethanol, $178 \mathrm{ppm}$ ethylacetate and $93 \mathrm{ppm}$ toluene in nitrogen, respectively, were observed. Recently, Penza et al. [32] applied a thin layer of SWCNT to the active area of a Love-mode SAW sensor built on $\mathrm{LiTaO}_{3} 36^{\circ} \mathrm{YX}$ substrate with a $\mathrm{ZnO}$ waveguide layer for various gases detection. They showed good linear relationship between the gas concentration and SAW phase change for the SAW sensors in response to $\mathrm{NO}_{2}, \mathrm{NH}_{3}$, and $\mathrm{H}_{2}$ with detection sensitivity of $3.3 \times 10^{-2}, 4.7 \times 10^{-2}$, and $1.8 \times 10^{-2} \mathrm{o} / \mathrm{ppm}$ respectively. One unique aspect of this work is that the authors also measured the change in electrical conductance of the SWCNT layer in response to gas exposure and matched the changes in electrical conductance and in SAW frequency shift nicely together in time. Such a correlated approach to multiple species detection may provide a unique capability to differentiate among various target species, a much-needed feature for a sensor that is responsive to multiple molecular species.

3.3. Standing Nanostructures. In addition to these random nanofibers, nanobelts, and nanotubes, vertical standing nanostructures such as nanowires, nanorods, and nanopillars have also been incorporated into the active surface of SAW sensors. For example, Arsat et al. [33] incorporated standing polyaniline nanowires on top of a $\mathrm{LiTaO}_{3} \mathrm{SAW}$ device for hydrogen detection. By varying the thickness of the polyaniline naniwire film they found that a thicker film led to a faster detection response. Sadek et al. [34] deposited standing $\mathrm{ZnO}$ nanorods onto the active surface of a SAW sensor fabricated on a $\mathrm{LiNbO}_{3} 64^{\circ} \mathrm{YX}$ substrate using a RF sputtering technique and used the SAW sensor for hydrogen detection. They demonstrated an optimum operating temperature range for the SAW sensor from 260 to $270^{\circ} \mathrm{C}$ with fairly good detection repeatability. Lately, more efforts were made to add $\mathrm{ZnO}$ nanorods to the active surface of SAW sensors. Wang et al. [35] fabricated a two-port SAW sensor incorporated with $\mathrm{ZnO}$ nanorods for UV detection. Huang et al. [36] from the same group pushed their effort one step further by adding $\mathrm{Pt}$ coated $\mathrm{ZnO}$ nanorods to a SAW sensor and used it for hydrogen detection.

Again as in those nanofiber and nanotube cases, the standing nanorods incorporated into the SAW sensors were mainly for rendering the SAW sensors responsive to their intended targets rather than for enhancing the sensing performances of the SAW sensors. A recent report shed some new light on this regard. Water and Chen [37] used a hydrothermal technique to add standing $\mathrm{ZnO}$ nanorods onto the active area of a Love-mode SAW sensor fabricated on a ST-cut Quartz substrate with an RF-sputtered $\mathrm{ZnO}$ waveguide layer. They reported an interesting finding that the detection sensitivity increases as the height of the nanorods increases when the ratio of nanorod height to wavelength is less or equal to 0.014 ; beyond this ratio the sensitivity decreases as the nanorod height increases until reaching zero when the ratio reaches 0.027 .

3.4. Porous Nanostructures. Sato et al. [38] reported the use of a SAW device coated with nanoporous anodized alumina as a humidity sensor. They found that a SAW device coated with a $1.0 \mu \mathrm{m}$ thick nanoporous alumina film yielded a much higher sensitivity when compared with a polymide film coated SAW device. The transient response of the SAW device with nanoporous film was one order faster than that of the polymide-coated SAW device. Hohkawa et al. [39] observed that SAW sensors incorporated with porous anodized alumina coated with platinum or cobalt were sensitive to ammonia. Varghese et al. [40] reported the detection of ammonia using nanoporous alumina with pore size of approximately $43 \mathrm{~nm}$ using a SAW sensor at room temperature. At a resonant frequency of $98.5 \mathrm{MHz}$ a linear relationship was established between ammonia concentration and frequency shift. Moreover, these nanoporous alumina enhanced SAW sensors were capable of measuring both low and high concentrations of ammonia. This is a remarkable improvement when compared with ammonia SAW sensors based on L-glutamic acid hydrochloride [41] where the sensors saturated quickly.

Siegal et al. [42, 43] used nanoporous carbon (NPC) film coated SAW sensors to detect a range of gases such as carbon tetrachloride, benzene, and trichloroethylene and achieved sensitivity values of 1070, 698, and $662 \mathrm{ppm}$, respectively. These sensitivity values are significantly higher when compared with the responses of dendrimer polymer coated SAW devices. In the studies, they also compared the response of siloxane coated SAW devices against NPC film coated SAW devices in detecting toluene, methanol and acetone. The NPC coated SAW devices showed much higher 
TABLE 2: Material constants for gold and PMMA.

\begin{tabular}{lcc}
\hline & Gold & PMMA \\
\hline$E$ & $79 \mathrm{GPa}$ & $3 \mathrm{GPa}$ \\
$\nu$ & 0.42 & 0.40 \\
$\rho$ & $19300 \mathrm{~kg} \cdot \mathrm{m}^{-3}$ & $1100 \mathrm{~kg} \cdot \mathrm{m}^{-3}$ \\
\hline
\end{tabular}

responses than their counterparts. The extrapolation of their reported data suggests that with NPC films of appropriate density $\left(<1.0 \mathrm{~g} \cdot \mathrm{cm}^{-3}\right)$, acetone concentration of less than $1 \mathrm{ppb}$ could be achieved.

\section{A Computation Approach to Elucidating the Effect of Adding Skyscraper Nanopillars}

Most of the nanostructures used in the approaches discussed earlier are generally coated or deposited onto the active surface. Because of the limited size of the active area, these approaches have their limitation in terms of how much the active surface area can be enhanced. As discussed previously [18], we believe that the use of vertically standing nanostructures holds great promise to providing a highly enhanced active surface area. This is because by building up in a "skyscraper" metaphor a much larger surface area increase can be achieved within a limited footprint area. As illustrated in Figure 2(a), in which a hexagonal array of vertically aligned nanopillars is constructed on a planar substrate, for a case with $r=150 \mathrm{~nm}, h=6 \mu \mathrm{m}$ and $p=75 \%$ for the nanopillars (note, $p \leq 91 \%$ ), a surface area increase $\left(S / S_{0}\right.$, where $S$ is the enhanced area and $S_{0}$ is the footprint area) of 61 -fold is achieved. Therefore, adding 3D skyscraper nanopillars onto the active surface of a SAW sensor (see Figure 2(b)) will offer a significant increase in its overall active surface area. However, adding nanostructures to the active surface of a SAW sensor will change the surface morphology, which in turn would add scattering loss and affect profoundly the behavior of wave propagation. To elucidate this impact it is imperative that the effect of adding standing nanostructures on the wave propagation and detection sensitivity be investigated analytically.

Over the years, various analytical methods have been used to study the underlying mechanism for SAW propagation. Among these methods, the delta function model [44], equivalent network model [45], Green's function model [46, 47], and coupling-of-mode method [48] are most notable. For instance, the Green's function method was applied to determine material parameters such as elastic constants and density in a nanoscale TiN thin film [49]. Generally, these methods are able to address certain design issues associated with SAW devices, but they cannot predict the full-scale behavior of these SAW devices. In these models, the second-order effects such as backscattering, diffraction and mechanical loading have either been ignored or simplified, thus making it highly difficult to predict the behavior of the SAW devices for high-frequency applications where the second-order effects are significant.
Finite-element analysis (FEA) has been proven advantageous for both component level and systems level analyses of SAW devices. The frequency response characterization of SAW filters [50,51], the electrical parameter characterization of SAW devices [52, 53], and the simulation of a SAW hydrogen sensor [54] have all been analyzed using FEA. We thus believe that FEA is well suited for investigating the effect of changing morphology of the sensitive surface on the propagation of SAW and the detection performance of a SAW sensor.

\section{The Underlying Governing Equations for SAW Operation}

The propagation of acoustic waves in a piezoelectric material is governed by the following coupled electromechanical constitutive equations:

$$
\begin{aligned}
& T=C_{E} \bullet S-e^{t} \bullet E, \\
& d=e \bullet S+\varepsilon \bullet E,
\end{aligned}
$$

where $T$ is the stress tensor, $C_{E}$ the stiffness matrix, $S$ the strain tensor, $e$ the piezoelectric coupling tensor, $E$ the electric field vector, $d$ the electrical displacement, $\varepsilon$ the dielectric matrix, and the superscript $t$ represents the transpose of a matrix. These constitutive equations can be related to the applied electrical potential and the induced mechanical displacements by applying Newton's law for mechanical movements and Gauss's law for electrostatic movements. According to Newton's second law of motion, the stress can be expressed as $\nabla \cdot T=\rho \ddot{u}-F$, where $\rho$ is the density of the substrate material, $\ddot{u}$ is the particle acceleration, and $F$ is the mechanical force. Since there is no internal or external force acting on the substrate, this equation reduces to $\nabla \bullet T=\rho \ddot{u}$. Based on Gauss's law, the electrical displacement can be expressed as $\nabla \bullet d=0$ when the electrical charge density is zero. Moreover, in a linear material, the electrical displacement is directly proportional to the electric field $d=\varepsilon E$. This equation can be further written as $d=-\varepsilon \nabla \varphi$ after applying $E=-\nabla \varphi$, where $\varphi$ is the electric potential. Moreover, the linear straindisplacement relationship can be written as $S=(\nabla u+$ $\nabla \tilde{u}) / 2=\nabla_{s} u$.

With these relationships, the constitutive equation (1) can be expressed in terms of the applied potential $(\varphi)$ and the induced mechanical displacement and acceleration $(u$ and $\ddot{u})$ along with the material properties $\left(C_{E}, e^{t}\right.$, and $\left.\varepsilon\right)$ as

$$
\begin{gathered}
\nabla \bullet\left[e^{t} \bullet \nabla \varphi\right]+\nabla \bullet\left[C_{E} \bullet \nabla_{s} u\right]-\rho \ddot{u}=0 \\
\nabla \bullet\left[e^{t} \bullet \nabla_{s} u\right]=\nabla \bullet[\varepsilon \bullet(\nabla \varphi)]
\end{gathered}
$$

For an anisotropic piezoelectric material like the XY-lithiumniobate substrate, the stiffness matrix $\left(C_{E}\right)$, the piezoelectric 


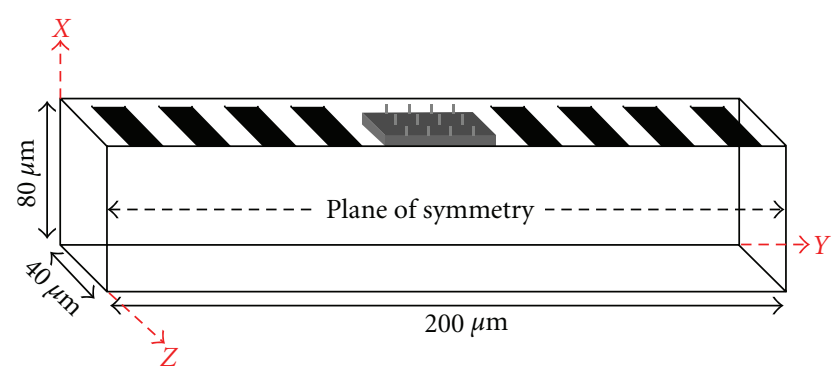

Figure 3: A computer model of a two-port delay-line SAW sensor with the active area incorporated with 12 evenly spaced standing nanopillars.

coupling matrix $(e)$, and the dielectric matrix $(\varepsilon)$ can be expressed as

$$
\begin{aligned}
C_{E} & =\left(\begin{array}{cccccc}
C_{11} & C_{12} & C_{13} & C_{14} & 0 & 0 \\
C_{12} & C_{11} & C_{13} & -C_{14} & 0 & 0 \\
C_{13} & C_{13} & C_{33} & 0 & 0 & 0 \\
C_{14} & -C_{14} & 0 & C_{44} & 0 & 0 \\
0 & 0 & 0 & 0 & C_{44} & C_{14} \\
0 & 0 & 0 & 0 & C_{14} & \frac{\left(C_{11}-C_{12}\right)}{2}
\end{array}\right), \\
e & =\left(\begin{array}{cccccc}
0 & 0 & 0 & 0 & e_{15} & -e_{22} \\
-e_{22} & e_{22} & 0 & e_{15} & 0 & 0 \\
e_{31} & e_{31} & e_{33} & 0 & 0 & 0
\end{array}\right), \\
& \varepsilon=\left(\begin{array}{ccc}
\varepsilon_{11} & 0 & 0 \\
0 & \varepsilon_{22} & 0 \\
0 & 0 & \varepsilon_{33}
\end{array}\right) .
\end{aligned}
$$

For an isotropic material such as gold and polymethylmethacrylate (PMMA), the stiffness matrix can be calculated from its Young's modulus $E$ and Poisson ratio $v$ as

$$
\begin{aligned}
C_{E} & =\frac{E}{(1+\nu)(1-2 v)} \\
& \times\left(\begin{array}{cccccc}
1-\nu & v & v & 0 & 0 & 0 \\
\nu & 1-\nu & v & 0 & 0 & 0 \\
\nu & \nu & 1-\nu & 0 & 0 & 0 \\
0 & 0 & 0 & \frac{(1-2 \nu)}{2} & 0 & 0 \\
0 & 0 & 0 & 0 & \frac{(1-2 \nu)}{2} & 0 \\
0 & 0 & 0 & 0 & 0 & \frac{(1-2 \nu)}{2}
\end{array}\right) .
\end{aligned}
$$

The piezoelectric coupling matrix of an isotropic material is zero and its dielectric matrix has a common constant value for the three elements along the principal diagonal line: $\varepsilon_{11}=$ $\varepsilon_{22}=\varepsilon_{33}=\varepsilon, \varepsilon=2.6$ for PMMA and $\varepsilon=6.9$ for gold.

\section{A Modeling Example and Some Selected Results}

Recently, we developed a finite element based computational method to investigate the SAW propagation behavior in nanostructure enhanced SAW biosensors $[55,56]$. A brief summary of the technical details of the modeling consideration is discussed here. A two-port delay-line SAW sensor having a flat sensitive surface with 12 nanopillars incorporated onto it is illustrated in Figure 3. Due to symmetry, only a half-structure model needs to be considered. For the SAW device, two sets of IDTs are placed $40 \mu \mathrm{m}$ apart: the one on the left is for generating the SAW and the one on the right is for receiving the SAW. Each of the two IDTs has four electrode fingers arranged in two alternating pairs, with the width and the spacing of the electrodes both set at $10 \mu \mathrm{m}$. The SAW sensor has a flat gold film with dimensions of $20 \mu \mathrm{m} \times 20 \mu \mathrm{m} \times 1 \mu \mathrm{m}$ placed in between the two IDTs. To enhance the sensing performance using nanostructures, square gold nanopillars having a width of $100 \mathrm{~nm}$ and a height varying from $100 \mathrm{~nm}$ up to $1 \mu \mathrm{m}$ are added to the flat gold film. For examining the detection performance of these SAW sensors, a thin film (100 nm) of PMMA is placed on top of the sensitive surface to simulate molecular adsorption.

For SAW generation, an impulse electrical signal (5) is applied to the electrode fingers of the generator IDTs. The generated wave travels to the right along the $\mathrm{Y}$ direction (note that the wave will actually travel in both directions and the left-traveling wave will be reflected back to the right after it reaches the left edge). After being interrogated by the PMMA film adsorbed on the sensitive surface, the acoustic wave is converted back to an electrical signal at the receiver IDTs. In the models, the following electrical boundary conditions are applied: zero charge/symmetry is applied to the top boundary surrounding the IDTs and to the symmetric plane, and ground condition is applied to all other boundaries. The impulse potential to the electrodes is applied in an alternating manner (i.e., $V_{i+}$ at the first and third electrodes, and $V_{i-}$ at the second and fourth electrodes):

$$
V_{i+}=\left\{\begin{array}{ll}
+0.5 V, & t \leq 1 \mathrm{~ns}, \\
0 V, & t \geq 1 \mathrm{~ns},
\end{array} \quad V_{i-}= \begin{cases}-0.5 V, & t \leq 1 \mathrm{~ns}, \\
0 V, & t \geq 1 \mathrm{~ns} .\end{cases}\right.
$$

The output voltage at the receiver IDTs is obtained in a similar alternating manner by measuring the differential potential between the first-third pair and the second-fourth pair of electrodes.

COMSOL Multiphysics (Burlington, MA) is used to find numerical solutions to the problem defined by the governing equation (2). The material constants listed in Tables 1 and $2[50,51]$ are used in the numerical analyses. In each case, signal attenuation due to PMMA adsorption at the sensitive surface is examined by quantifying the insertion loss (IL) characteristics using the following formula:

$$
\mathrm{IL}=-20 \times \log _{10}\left|\frac{V_{\text {output }}}{V_{\text {input }}}\right|
$$




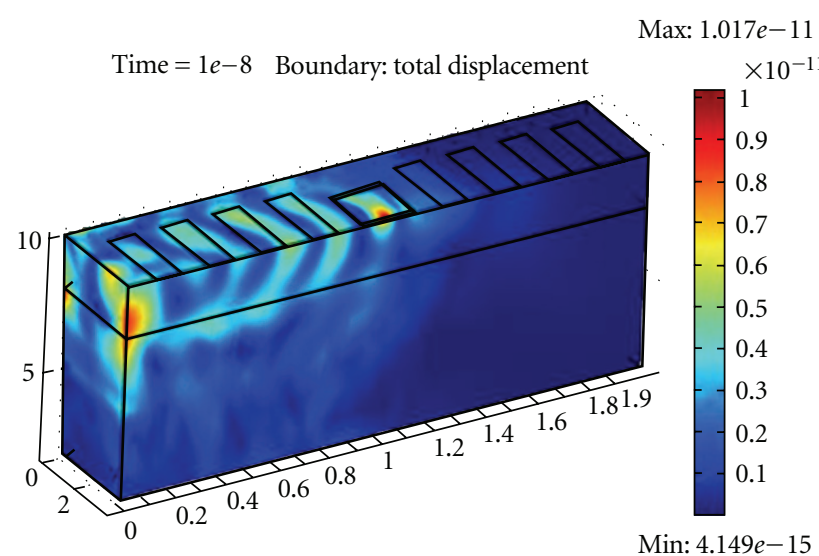

(a)

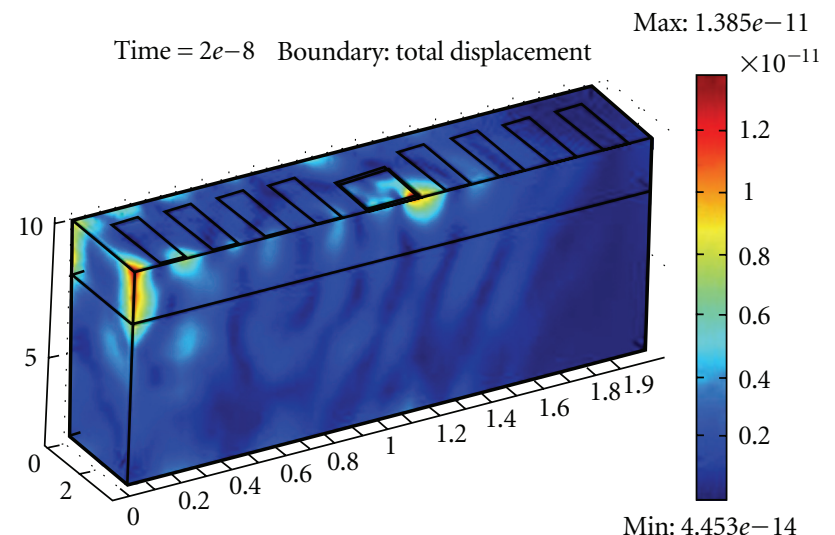

(b)

Figure 4: Snapshots of wave propagation from the SAW model captured at $10 \mathrm{~ns}$ (a) and at $20 \mathrm{~ns}$ (b) after the application of an impulse excitation.

Figure 4 shows two snap-shot images of wave propagation in the SAW sensor captured at $10 \mathrm{~ns}$ and $20 \mathrm{~ns}$, respectively, after the impulse potential is applied. These images reveal that the generated wave has traveled a distance of approximately $39 \mu \mathrm{m}$ away from the leading edge of the generator IDT in $10 \mathrm{~ns}$, and after $20 \mathrm{~ns}$ the wave reached the other side of the substrate. Based on the travel distance, the velocity of the acoustic wave is estimated to be $3900 \mathrm{~m} / \mathrm{s}$, which is close to the reported value (ranging from 3700 to $3750 \mathrm{~m} / \mathrm{s}$ ) for an acoustic wave traveling in a XY-lithiumniobate piezoelectric material [57]. Figure 5(a) shows the obtained IL spectra for a flat control case (i.e., a SAW sensor with a flat gold sensitive surface) before and after the adsorption of a $100 \mathrm{~nm}$ thick PMMA film, and Figures 5(b) and 5(c) show the IL spectra for the SAW sensors with 9 and 12 nanopillars, respectively, before and after the adsorption of a PMMA film of the same thickness. From these IL spectra, the resonant frequency for the flat control case is calculated to be $98.5 \mathrm{MHz}$. Based on the design, this SAW sensor is expected to have a resonant frequency of $f=97.5 \mathrm{MHz}$ which is reasonably close to the observed value. The slight difference observed here can be attributed to the interference

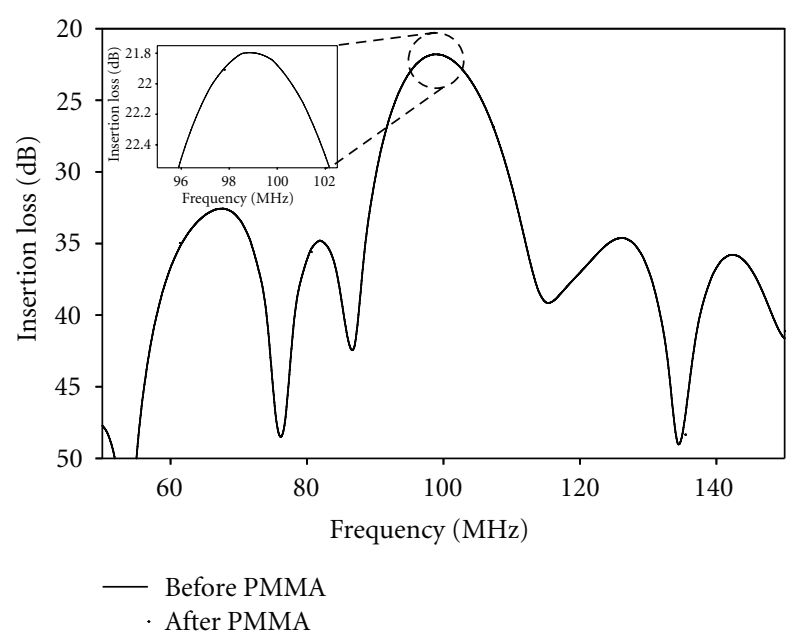

(a)

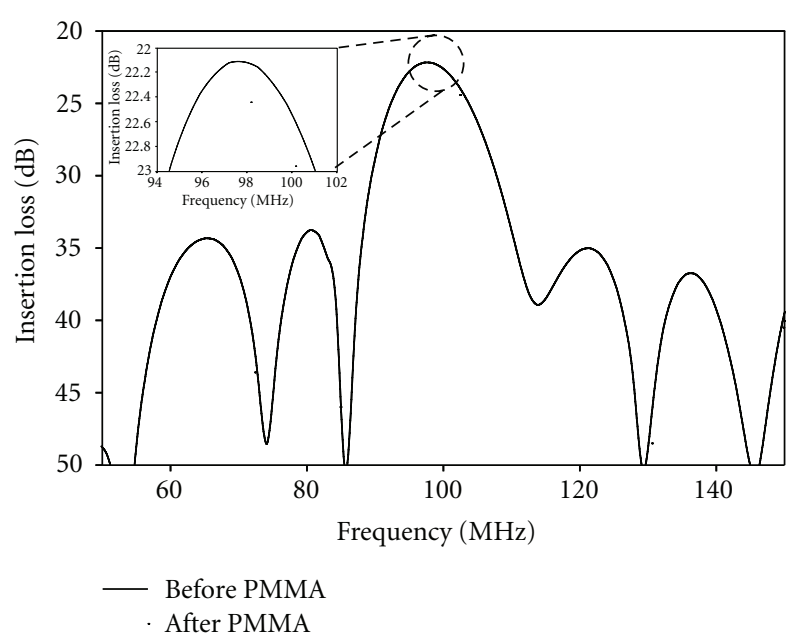

(b)

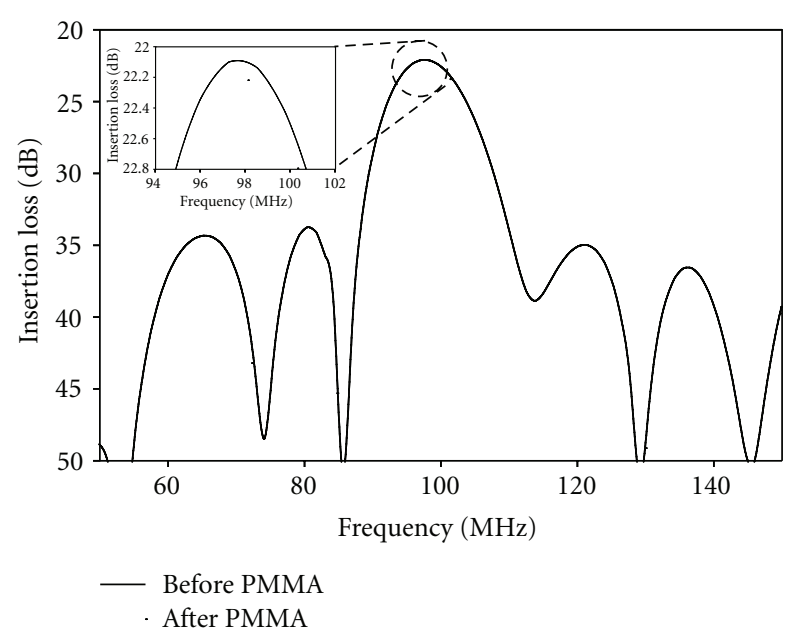

(c)

Figure 5: Insertion loss spectra for the flat control case (a), a nanocase with 9 nanopillars (b), and a nanocase with 12 nanopillars (c) with each insert showing a zoom-in view of the peak frequency. 


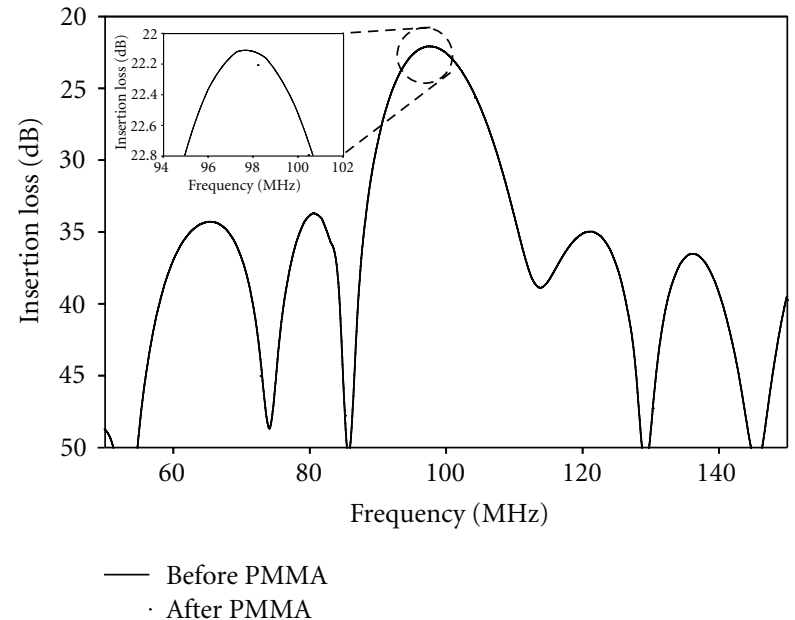

(a)

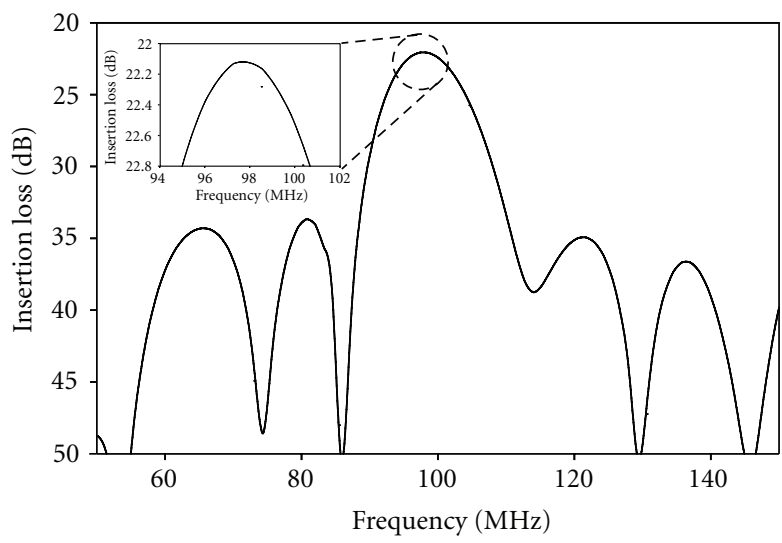

- Before PMMA After PMMA

(c)

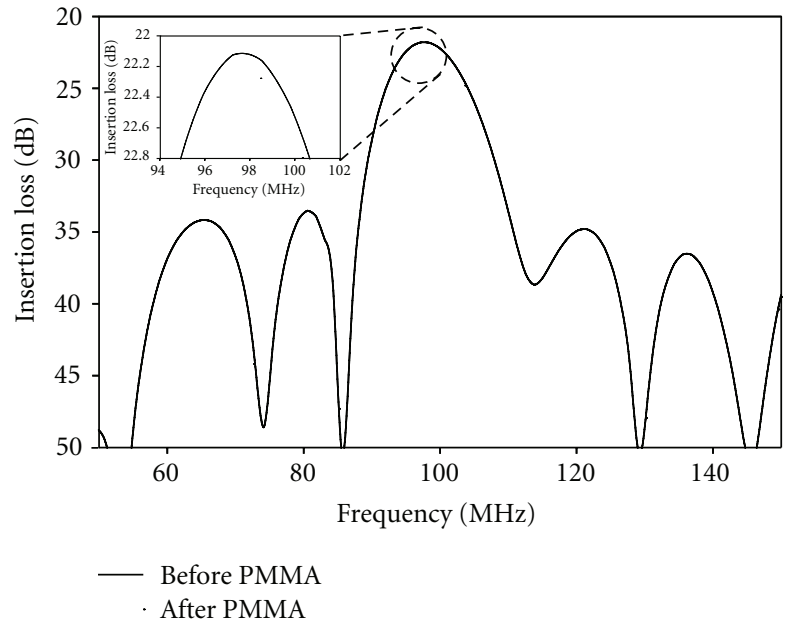

(b)

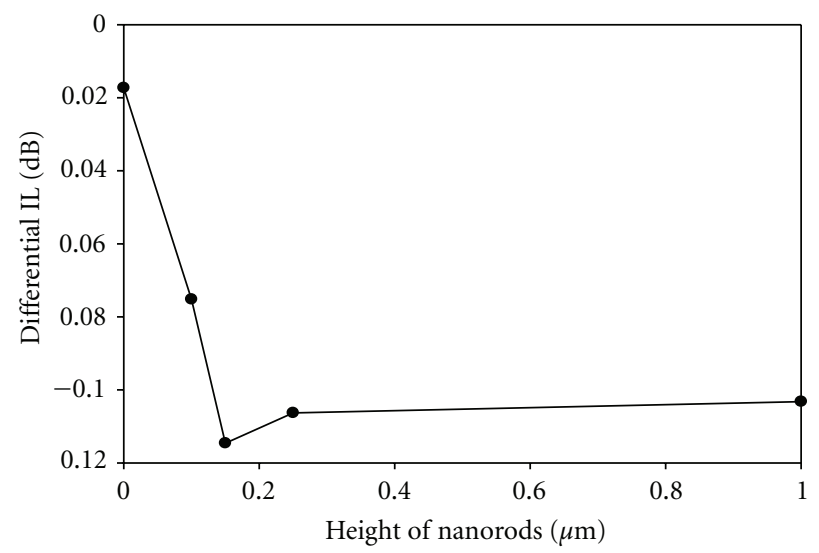

(d)

Figure 6: Insertion loss spectra for a SAW sensor with 9 nanopillars having a height of $100 \mathrm{~nm}$ (a), $150 \mathrm{~nm}$ (b), and $250 \mathrm{~nm}$ (c) before and after the adsorption of a $100 \mathrm{~nm}$ thick PMMA film with each insert showing a zoom-in view of the peak frequency. (d) Variation of the differential IL with the height of nanopillars.

of the reflected wave and the fact that only four individual electrodes are considered for the IDTs in the model whereas the theoretical value is predicted based on a much larger number of individual electrodes.

Higher IL is observed from simulation for a sensor with nanopillars than one without nanopillars. For instance, the peak IL $\mathrm{f}$ for the SAW sensor with a flat sensitive surface is $21.802 \mathrm{~dB}$, but the peak IL for the SAW sensors with 9 nanopillars and 12 nanopillars is $22.103 \mathrm{~dB}$ and $22.123 \mathrm{~dB}$, respectively, before the adsorption of the PMMA film. After the adsorption of the PMMA film, the peak IL increases to $21.820 \mathrm{~dB}$ (flat), $22.206 \mathrm{~dB}$ (9-nanopillar), $22.421 \mathrm{~dB}$ (12nanopillar) for the respective SAW sensors. By taking the ratio of the differential IL measurement to the actual mass of the adsorbed PMMA, the detection sensitivity of a SAW sensor is quantified. The actual mass of the adsorbed PMMA is calculated by multiplying the volume of the adsorbed
PMMA in each case by the density $\rho=1000 \mathrm{~kg} \cdot \mathrm{m}^{-3}$ of PMMA material. The detection sensitivity for the flat control case is calculated as $0.345 \mathrm{~dB} / \mathrm{ng}$ while the detection sensitivity for the SAW sensors with 9 and 12 nanopillars are $1.94 \mathrm{~dB} / \mathrm{ng}$ (5.62 times higher than the flat control case) and $5.85 \mathrm{~dB} / \mathrm{ng}$ (16.96 times higher than the flat control case), respectively. Thus, the addition of vertically standing nanopillars to the flat sensitive surface is responsible for the increase in the total surface area for molecular adsorption, thus leading to increased detection sensitivity.

Figures 6(a)-6(c) show the IL spectra for a SAW sensor with 9 nanopillars having different pillar heights before and after the adsorption of the $100 \mathrm{~nm}$ thick PMMA film. The obtained peak IL measures $22.125 \mathrm{~dB}, 22.128 \mathrm{~dB}$ and $22.105 \mathrm{~dB}$, respectively, when the nanopillars are $100 \mathrm{~nm}$, $150 \mathrm{~nm}$ and $250 \mathrm{~nm}$ tall before the adsorption of the PMMA film. After the adsorption of the PMMA film, the peak 
IL values increases to $22.200 \mathrm{~dB}, 22.243 \mathrm{~dB}$, and $22.211 \mathrm{~dB}$, respectively. Figure $6(\mathrm{~d})$ shows the variation of the differential peak IL (due to the adsorption of the PMMA film) with the nanopillar height. With nanopillar heights being $100 \mathrm{~nm}$, $150 \mathrm{~nm}, 250 \mathrm{~nm}$, and $1 \mu \mathrm{m}$, a differential peak IL of $0.075 \mathrm{~dB}$, $0.115 \mathrm{~dB}, 0.106 \mathrm{~dB}$, and $0.103 \mathrm{~dB}$ is measured, respectively. The differential peak IL increases significantly when the height of nanopillars increases from zero to $150 \mathrm{~nm}$, and beyond this height the differential peak IL decreases slightly as the height of nanopillars increases. This fact suggests that it may not be beneficial to have nanopillars taller than $150 \mathrm{~nm}$. Moreover, this result is consistent with the observation made by Water and Chen [37] from their experimental work.

\section{Summary}

Love-mode SAW biosensors hold great promise to deliver high sensitivity and wireless and remote operational capabilities that are ideal for many biological applications. Although many parameters such as waveguide thickness, substrate material, and waveguide material have been explored in efforts to improve the performance of SAW-based biosensors, there is a technological disjunction that hinders the application of SAW sensors for highly demanding biological detection needs. To address this issue, one solution is to incorporate vertically aligned, optimally spaced and mechanically robust nanostuctures into the active area of the SAW biosensors. Based on a computational modeling analysis, it is clear that adding standing nanopillars to the sensitive surface does provide the desired benefits: it provides increased surface area for increased molecular adsorption, thus leading to increased attenuation per unit thickness of molecular adsorption. In general, increasing the number and the height of nanopillars will increase the detection sensitivity of the SAW sensors, but such an increase due to nanopillar height will reach a limit.

\section{Acknowledgments}

I would like to thank my student Yeswanth L. Rao for his contribution to the modeling work summarized here that he had done during his doctoral study at the University of Georgia. I would also thank the financial supports from the National Science Foundation, the Faculty of Engineering at the University of Georgia, and the Institute for Biological Interfaces of Engineering at Clemson University.

\section{References}

[1] G. Zhang, "Nanotechnology-based biosensors in drug delivery," in Nanotechnology in Drug Delivery, M. M. de Villiers, et al., Ed., vol. 163, chapter 6, Springer, New York, NY, USA, 2009.

[2] C. K. Campbell, Surface Acoustic Wave Devices: For Mobile and Wireless Communication, Academic Press, San Diego, Calif, USA, 1998.

[3] M. D. Ward and D. A. Buttry, "Insitu interfacial mass detection with piezoelectric transducers," Science, vol. 249, pp. 1000 1007, 1990.
[4] E. Gizeli, N. J. Goddard, C. R. Lowe, and A. C. Stevenson, "A love plate biosensor utilising a polymer layer," Sensors and Actuators B, vol. 6, no. 1-3, pp. 131-137, 1992.

[5] G. L. Harding, J. Du, P. R. Dencher, D. Barnett, and E. Howe, "Love wave acoustic immunosensor operating in liquid," Sensors and Actuators A, vol. 61, no. 1-3, pp. 279-286, 1997.

[6] T. Wessa, M. Rapp, and H. J. Ache, "New immobilization method for SAW-biosensors: covalent attachment of antibodies via CNBr," Biosensors and Bioelectronics, vol. 14, no. 1, pp. 93-98, 1999.

[7] M. J. Fernández, J. L. Fontecha, I. Sayago, et al., "Discrimination of volatile compounds through an electronic nose based on ZnO SAW sensors," Sensors and Actuators B, vol. 127, no. 1, pp. 277-283, 2007.

[8] B.-S. Joo, J.-S. Huh, and D.-D. Lee, "Fabrication of polymer SAW sensor array to classify chemical warfare agents," Sensors and Actuators B, vol. 121, no. 1, pp. 47-53, 2007.

[9] J. Andrä, A. Böhling, T. M. A. Gronewold, U. Schlecht, M. Perpeet, and T. Gutsmann, "Surface acoustic wave biosensor as a tool to study the interaction of antimicrobial peptides with phospholipid and lipopolysaccharide model membranes," Langmuir, vol. 24, no. 16, pp. 9148-9153, 2008.

[10] G. Treitz, T. M. A. Gronewold, E. Quandt, and M. ZabeKühn, "Combination of a SAW-biosensor with MALDI mass spectrometric analysis," Biosensors and Bioelectronics, vol. 23, no. 10, pp. 1496-1502, 2008.

[11] M. Thompson and D. C. Stone, Surface Launched Acoustic Wave Sensors: Chemical Sensing and Thin-Film Characterization, John Wiley \& Sons, New York, NY, USA, 1997.

[12] D. S. Ballantine, R. M. White, S. J. Martin, et al., Acoustic Wave Sensors: Theory, Design, and Physio-Chemical Applications, Academic Press, San Diego, Calif, USA, 1997.

[13] J. W. Gardner, V. K. Varadan, and O. O. Awadelkarim, Microsensors, MEMS, and Smart Devices, John Wiley \& Sons, New York, NY, USA, 2001.

[14] K. Länge, B. E. Rapp, and M. Rapp, "Surface acoustic wave biosensors: a review," Analytical and Bioanalytical Chemistry, vol. 391, no. 5, pp. 1509-1519, 2008.

[15] S. J. Ippolito, A. Trinchi, D. A. Powell, and W. Wlodarski, "Acoustic wave gas and vapor sensor in Solid State Sensoing," E. Comini, et al., Ed., chapter 8, Springer, New York, NY, USA, 2009.

[16] B. A. Auld, Acoustic Fields and Waves in Solids II, Kreiger, Malabar, Fla, USA, 1990.

[17] A. E. H. Love, Some Problems of Geodynamics, Cambridge University Press, Cambridge, UK, 1911.

[18] Y. L. Rao and G. Zhang, "Enhancing the sensitivity of SAW sensors with nanostructures," Current Nanoscience, vol. 2, no. 4, pp. 311-318, 2006.

[19] N. Levit, D. Pestov, and G. Tepper, "High surface area polymer coatings for SAW-based chemical sensor applications," Sensors and Actuators B, vol. 82, no. 2-3, pp. 241-249, 2002.

[20] C.-S. Chiu and S. Gwo, "Quantitative surface acoustic wave detection based on colloidal gold nanoparticles and their bioconjugates," Analytical Chemistry, vol. 80, no. 9, pp. 33183326, 2008.

[21] C.-S. Chiu, H.-M. Lee, C.-T. Kuo, and S. Gwo, "Immobilization of DNA-Au nanoparticles on aminosilane-functionalized aluminum nitride epitaxial films for surface acoustic wave sensing," Applied Physics Letters, vol. 93, no. 16, Article ID 163106, 2008. 
[22] A. Z. Sadek, D. Buso, A. Martucci, P. Mulvaney, W. Wlodarski, and K. Kalantar-zadeh, "Titanium dioxide-based $64^{\circ}$ YX $\mathrm{LiNbO}_{3}$ surface acoustic wave hydrogen gas sensors," Journal of Sensors, vol. 2008, Article ID 254283, 5 pages, 2008.

[23] T.-T. Wu, Y.-Y. Chen, and T.-H. Chou, "A high sensitivity nanomaterial based SAW humidity sensor," Journal of Physics $D$, vol. 41, no. 8, Article ID 085101, 2008.

[24] A. Z. Sadek, W. Wlodarski, K. Shin, R. B. Kaner, and K. Kalantar-zadeh, "A layered surface acoustic wave gas sensor based on a polyaniline/ $\mathrm{In}_{2} \mathrm{O}_{3}$ nanofibre composite," Nanotechnology, vol. 17, no. 17, pp. 4488-4492, 2006.

[25] A. Z. Sadek, W. Wlodarski, K. Shin, R. B. Kaner, and K. Kalantar-zadeh, "A polyaniline $/ \mathrm{WO}_{3}$ nanofiber compositebased $\mathrm{ZnO} / 64^{\circ} \mathrm{YX} \mathrm{LiNbO}_{3} \mathrm{SAW}$ hydrogen gas sensor," Synthetic Metals, vol. 158, no. 1-2, pp. 29-32, 2008.

[26] M. Z. Atashbar, A. Z. Sadek, W. Wlodarski, et al., "Layered SAW gas sensor based on CSA synthesized polyaniline nanofiber on $\mathrm{AlN}$ on $64^{\circ} \mathrm{YX} \mathrm{LiNbO}_{3}$ for $\mathrm{H}_{2}$ sensing," Sensors and Actuators B, vol. 138, no. 1, pp. 85-89, 2009.

[27] L. Al-Mashat, H. D. Tran, W. Wlodarski, R. B. Kaner, and K. Kalantar-zadeh, "Polypyrrole nanofiber surface acoustic wave gas sensors," Sensors and Actuators B, vol. 134, no. 2, pp. 826$831,2008$.

[28] A. Z. Sadek, W. Wlodarski, K. Kalantar-zadeh, et al., " $\mathrm{H}_{2}$ and $\mathrm{NO}_{2}$ gas sensors with $\mathrm{ZnO}$ nanobelt layer on $36^{\circ} \mathrm{LiTaO}_{3}$ and $64^{\circ} \mathrm{LiNbO}_{3} \mathrm{SAW}$ transducers," Proceedings of IEEE Sensors, vol. 2005, pp. 1343-1346, 2005.

[29] M. Penza, F. Antolini, and M. V. Antisari, "Carbon nanotubes as SAW chemical sensors materials," Sensors and Actuators B, vol. 100, no. 1-2, pp. 47-59, 2004.

[30] M. Penza, F. Antolini, and M. V. Antisari, "Carbon nanotubesbased surface acoustic waves oscillating sensors for vapor detection," Thin Solid Films, vol. 472, p. 246, 2005.

[31] M. Penza, G. Cassano, P. Aversa, et al., "Carbon nanotubescoated multi-transducing sensors for VOCs detection," Sensors and Actuators B, vol. 111-112, pp. 171-180, 2005.

[32] M. Penza, P. Aversa, G. Cassano, W. Wlodarski, and K. Kalantar-zadeh, "Layered SAW gas sensor with single-walled carbon nanotube-based nanocomposite coating," Sensors and Actuators B, vol. 127, no. 1, pp. 168-178, 2007.

[33] R. Arsat, X. F. Yu, Y. X. Li, W. Wlodarski, and K. Kalantarzadeh, "Hydrogen gas sensor based on highly ordered polyaniline nanofibers," Sensors and Actuators B, vol. 137, no. 2, pp. 529-532, 2009.

[34] A. Z. Sadek, W. Wlodarski, Y. X. Li, et al., "A ZnO nanorod based layered $\mathrm{ZnO} / 64^{\circ} \mathrm{YX} \mathrm{LiNbO}_{3} \mathrm{SAW}$ hydrogen gas sensor," Thin Solid Films, vol. 515, no. 24, pp. 8705-8708, 2007.

[35] W.-S. Wang, T.-T. Wu, T.-H. Chou, and Y.-Y. Chen, "A $\mathrm{ZnO}$ nanorod-based SAW oscillator system for ultraviolet detection," Nanotechnology, vol. 20, no. 13, Article ID 135503, 2009.

[36] F.-C. Huang, Y.-Y. Chen, and T.-T. Wu, "A room temperature surface acoustic wave hydrogen sensor with Pt coated $\mathrm{ZnO}$ nanorods," Nanotechnology, vol. 20, no. 6, Article ID 065501, 2009.

[37] W. Water and S.-E. Chen, "Using ZnO nanorods to enhance sensitivity of liquid sensor," Sensors and Actuators B, vol. 136, no. 2, pp. 371-375, 2009.

[38] M. Sato, T. Yamamoto, T. Meguro, and K. Yamanouchi, "Sensitivity of an anadically oxidized aluminium film on a surface acoustic wave sensor to humidity," Sensors and Actuators: B, vol. 20, no. 2-3, pp. 205-212, 1994.

[39] K. Hohkawa, K. Komine, H. Suzuki, T. Eguchi, Y. Sato, and K. Koh, "Piezoelectric sensor using a porous alumina film covered with a catalytic material," in Proceedings of IEEE Ultrasonics Symposium, vol. 1, pp. 513-516, 1998.

[40] O. K. Varghese, D. Gong, W. R. Dreschel, K. G. Ong, and C. A. Grimes, "Ammonia detection using nanoporous alumina resistive and surface acoustic wave sensors," Sensors and Actuators B, vol. 94, no. 1, pp. 27-35, 2003.

[41] C.-Y. Shen, C.-P. Huang, and H.-C. Chuo, "The improved ammonia gas sensors constructed by L-glutamic acid hydrochloride on surface acoustic wave devices," Sensors and Actuators B, vol. 84, no. 2-3, pp. 231-236, 2002.

[42] M. P. Siegal, D. L. Overmyer, R. J. Kottenstette, D. R. Tallant, and W. G. Yelton, "Nanoporous-carbon films for microsensor preconcentrators," Applied Physics Letters, vol. 80, no. 21, p. 3940, 2002.

[43] M. P. Siegal, W. G. Yelton, D. L. Overmyer, and P. P. Provencio, "Nanoporous carbon films for gas microsensors," Langmuir, vol. 20, no. 4, pp. 1194-1198, 2004.

[44] R. H. Tancrell and M. G. Holland, "Acoustic surface wave filters," Proceedings of IEEE, vol. 59, no. 3, p. 393, 1971.

[45] W. R. Smith, H. M. Gerals, J. H. Collins, T. M. Reeder, and H. J. Shaw, "Analysis of interdigitated surface wave transducers by use of equivalent circuit model," IEE Transactions of Microwave Theory \& Techniques, vol. 17, no. 11, p. 856, 1969.

[46] F. Huang and E. G. S. Paige, "The scattering of surface acoustic waves by electrical effects in two-dimensional metal film structures," IEEE Transactions on Ultrasonics, Ferroelectrics, and Frequency Control, vol. 35, no. 6, pp. 723-735, 1988.

[47] D. Qiao, W. Liu, and P. M. Smith, “General Green's functions for SAW device analysis," IEEE Transactions on Ultrasonics, Ferroelectrics, and Frequency Control, vol. 46, no. 5, pp. 12421253, 1999.

[48] E. Akcakaya, "A new analysis of single phase unidirectional transducers," IEEE Transactions on Ultrasonics, Ferroelectrics, and Frequency Control, vol. 34, no. 1, pp. 45-52, 1987.

[49] V. K. Tewary, “Green's-function method for modeling surface acoustic wave dispersion in anisotropic material systems and determination of material parameters," Wave Motion, vol. 40, no. 4, pp. 399-412, 2004.

[50] G. Xu, "Direct finite-element analysis of the frequency response of a Y-Z lithium niobate SAW filter," Smart Materials and Structures, vol. 9, no. 6, pp. 973-980, 2000.

[51] G. Xu and Q. Jiang, "A finite element analysis of second order effects on the frequency response of a SAW device," Journal of Intelligent Material Systems and Structures, vol. 12, no. 2, pp. 69-77, 2001.

[52] S. J. Ippolito, K. Kalantar-zhadeh, D. A. Powell, and W. Wlodarski, "A finite element approach for 3-dimensional simulation of layered acoustic wave transducers," in Proceedings of IEEE Conference on Optoelectronic and Microelectronic Materials and Devices, p. 541, 2001.

[53] S. J. Ippolito, K. Kalantar-zadeh, D. A. Powell, and W. Wlodarski, "A 3-dimensional finite element approach for simulating acoustic wave propagation in layered SAW devices," in Proceedings of IEEE Ultrasonics Symposium, vol. 1, pp. 303306, 2003.

[54] M. Z. Atashbar, B. J. Bazuin, M. Simpeh, and S. Krishnamurthy, "3D FE simulation of $\mathrm{H}_{2}$ SAW gas sensor," Sensors and Actuators B, vol. 111-112, pp. 213-218, 2005.

[55] Y. L. Rao and G. Zhang, "3D modeling of a surface-acousticwave based sensor," in Proceedings of COMSOL Conference, $\mathrm{p}$. 89, 2007. 
[56] Y. L. Rao, Nanostructure-enhanced surface acoustic Love wave devices for biosensing application, Ph.D. dissertation, The University of Georgia, Athens, Ga, USA, 2008.

[57] B. Lin, G. Chen, X. R. Zhang, D. Zhang, and J. C. Chen, "Calculation of the SAW velocity change of proton exchanged $\mathrm{LiNbO}_{3}$ crystal," in Proceedings of the American Institute of Physics, vol. 657, p. 1284, 2003. 

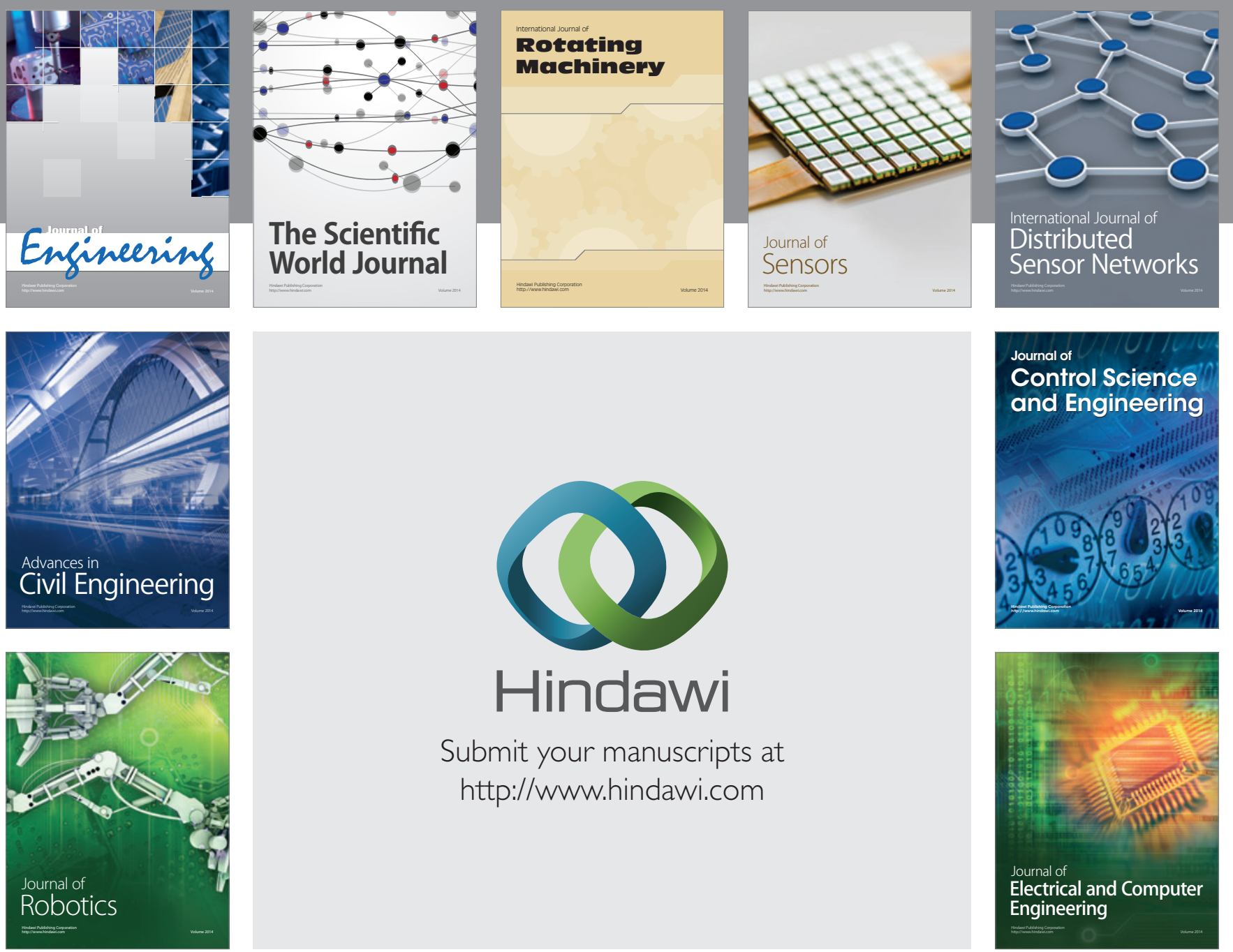

Submit your manuscripts at

http://www.hindawi.com
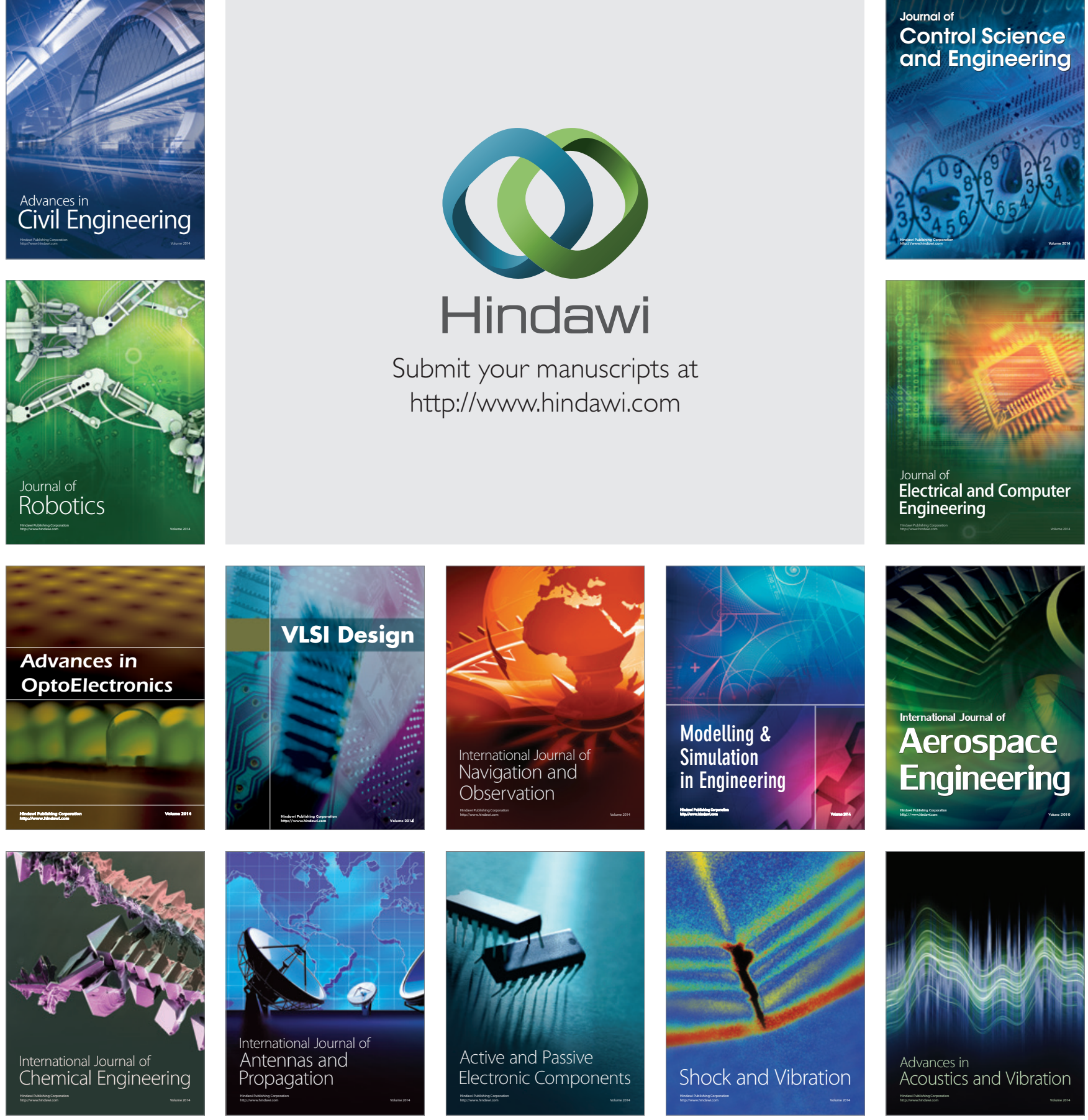